\title{
Évaluation de la diversité et de la charge parasitaire des lacs de la ville de Yamoussoukro en Côte d'Ivoire
}

\author{
SYLLA Idrissa ${ }^{1 *}$, KOFFI Mathurin ${ }^{1}$, N'DRI Kouamé Marius ${ }^{1}$, BONY Kotchi Yves ${ }^{1}$, ASSEMIAN \\ N'Guessan Emmanuel ${ }^{1}$, KONAN Koffi Félix ${ }^{1}$, NIAMIEN-EBROTTIE Julie ${ }^{2}$ et EDIA Oi Edia ${ }^{2}$ \\ 1. Unité de Formation et de Recherche Environnement, Université Jean Lorougnon GUEDE, BP 150 Daloa, Cote \\ d'Ivoire \\ 2. Laboratoire d'Environnement et de Biologie Aquatique, U.F.R.-S.G.E., Université Nangui Abrogoua, 02 BP 801 \\ Abidjan 02, Cote d'Ivoire. \\ *E-mail de l'auteur de la correspondance : syllaidris@gmail.com \\ Original submitted in on $18^{\text {th }}$ January 2019. Published online at www.m.elewa.org/journals/ on $28^{\text {th }}$ February 2019 \\ https://dx.doi.org/10.4314/jab.v134i1.2
}

\section{RÉSUMÉ :}

Objectif : Cette étude vise à caractériser les parasites des eaux des lacs de Yamoussoukro et d'étudier les risques sanitaires dans la réutilisation de ces eaux usées afin d'en tirer les conséquences épidémiologiques.

Méthodologie et résultats : Vingt-huit échantillons d'eaux résiduaires ont été collectés dans huit lacs de Yamoussoukro à partir de récipients stériles. La méthode de Bailenger a été utilisée pour la mise en évidence des parasites. La reconnaissance spécifique et le dénombrement des parasites ont été faits à l'aide d'un microscope optique. Les prévalences observées ont été comparées entre lacs et par espèce de parasite.

Au total, 13 espèces de parasites: Ankylostoma sp., Strongyloïde sp., Ascaris sp., Nematodirus sp., Trichuris sp., Toxocara sp., Capillaria sp., Ascaridia galli, Enterobius vermicularis, Taenia sp., Hymenolepis diminuta., Entamoeba coli., Entamoeba histolytica et les larves de Strongles ont été détectées dans les eaux des huit lacs investigués à Yamoussoukro. Les œufs d'helminthes sont quasi-présents dans tous les lacs investigués avec des charges parasitaires variables. Trois espèces ont donné des charges parasitaires très élevées : L'espèce Ankylostoma sp a été retrouvée dans tous les lacs. Les Strongyloïdes et les Ascaris sp présentaient des prévalences de $100 \%$ et $87,5 \%$ respectivement. Au niveau des lacs investigués, lacs, le lac $\mathrm{N}^{\circ} 5$ était le plus parasité avec une prévalence de $100 \%$ sur l'ensemble des parasites, ensuite viennent les lacs $N^{\circ} 1,6$ et $8(69,2 \%)$ puis les lacs G0, 9 et 10 (53,8\%).

Conclusion et application des résultats: Les analyses parasitologiques montrent que les lacs de Yamoussoukro sont contaminés par des parasites d'importances médicales. L'infestation de ces eaux en agents pathogènes interdit leur usage en agriculture ce qui n'est pas couramment observé sur le terrain.

Mots clés : Parasites, lacs, eaux résiduaires, Yamoussoukro, Côte d'Ivoire 


\section{ABSTRACT}

Objectives: Water, source of life is essential for all living beings. After its use, the water is loaded in various elements modifying its physical, chemical and biological characteristics. One of the most serious environmental problems is the problem of sanitation. Indeed, human activity leads to the production of wastewater discharged daily into the wild.

Methodology and results: Twenty-eight waste water samples were collected from eight Yamoussoukro lakes from sterile containers. The method of Bailenger was used for the detection of parasites. The specific recognition and enumeration of the parasites was done using an optical microscope Prevalences observed were compared between lakes and by parasite species. A total of 13 species of parasites: Ankylostoma sp., Strongyloïd sp., Ascaris sp., Nematodirus sp., Trichuris sp., Toxocara sp., Capillaria sp., Ascaridia galli, Enterobius vermicularis, Taenia sp., Hymenolepis diminuta., Entamoeba coli., Entamoeba histolytica et les larves de Strongles were detected in the waters of Yamoussoukro lakes. Helminth eggs are almost present in all investigated lakes with variable parasite loads. Three species gave very high parasite loads: The species Ankylostoma sp was found in all lakes. Strongyloids and Ascaris sp had prevalence rates of $100 \%$ and $87.5 \%$, respectively. At the level of the parasite load of the lakes, Lake ${ }^{\circ} 5$ was the most parasitized with a prevalence of $100 \%$ on all the parasites, then the lakes $N^{\circ} 1,6$ and $8(69,2 \%)$ then the Lakes $\mathrm{G} 0$, 9 and $10(53.8 \%)$.

Conclusion and application of results: Parasitological analysis show that Yamoussoukro lakes are contaminated by parasites of medical importance. The infestation of these waters with pathogens prohibits their use in agriculture, which is not commonly observed in the field.

Keywords: Parasites, lakes, wastewater, Yamoussoukro, Côte d'Ivoire

\section{INTRODUCTION}

Ces dernières décennies, une attention croissante de la communauté scientifique s'est portée sur la préservation de l'environnement. Parmi les problèmes d'environnement les plus préoccupants, figure en bonne place le problème de l'assainissement de façon générale et en particulier celui des eaux usées (Akpo et al., 2013). En effet, l'activité humaine entraîne la production d'eaux usées déchargées quotidiennement dans la nature. Ce rejet d'eaux usées brutes dans les milieux naturels est une pratique courante dans la plupart des pays en développement. Cette situation est accentuée dans les centres urbains du fait de la démographie galopante qui les caractérise. Les modifications majeures observées, dues tant aux activités anthropiques (industrialisation et urbanisation rapides) qu'aux facteurs naturels, ont potentiellement des conséquences sanitaires et environnementales (Strobl et Robillard, 2008). Les effluents urbains constituent une source majeure de polluants et de contaminants parasitaires dans l'eau. L'eau, source de vie qui est essentielle pour de multiples usages (domestiques, industriels et agricoles) devient ainsi un danger pour l'homme et les animaux. Par ailleurs, sa qualité devient un facteur influençant l'état de santé, la morbidité et la mortalité à la fois chez l'homme et les animaux (Kazi et al., 2009). Les chiffres publiés par l'Organisation Mondiale de la Santé (OMS) en 2004 révèlent que chaque année 1,8 million de personnes dont $90 \%$ d'enfants de moins de cinq ans, vivant pour la plupart dans les pays en développement, meurent de maladies diarrhéiques. Or, à l'échelle mondiale, $88 \%$ des maladies diarrhéiques sont imputables à la mauvaise qualité de l'eau de boisson et à un assainissement insuffisant des eaux usées (Attab, 2011). Au vu des chiffres qui précèdent, l'eau est devenue aujourd'hui un enjeu stratégique mondial dont la gestion doit impérativement s'intégrer dans une politique de développement durable. Pourtant, dans la plupart des pays en développement, par manque de moyens, le contrôle et le suivi de la qualité des eaux résiduaires réutilisées ne sont pas systématiques (Sadat et al., 2011). En Afrique, c'est généralement en zones arides (zone sahélienne) que le contrôle et l'épuration des eaux 
usées avant leur réutilisation est très pratiquée. Ceci à cause des ressources en eau limitées, contrairement aux zones tropicales humides où le traitement des eaux usées n'est pas une pratique prioritaire à cause de la pluviométrie qui est relativement abondante et donc moins d'études sont orientées vers ce domaine (Amy et al., 1996). Cependant, ces eaux usées sont couramment réutilisées dans certains domaines notamment en agriculture. C'est le cas des populations de la ville de Yamoussoukro, la capitale de la Côte d'Ivoire qui, en raison de la surpopulation et de la méconnaissance ou de la négligence des règles d'hygiène, rejettent les eaux usées directement dans les lacs. En effet, ces lacs mis en place dans l'optique de rendre la ville de Yamoussoukro rayonnante d'aspect touristique sont devenus aujourd'hui, en plus des lieux de dépôt d'ordures de tous genres, la cible d'évacuation des eaux usées brutes collectées des domiciles, des industries, des centres de santé et de l'eau de ruissellement à travers des caniveaux et égouts. Ces lacs sont couverts de végétaux aquatiques à plusieurs endroits, sans oublier la présence des lavandiers et de stations de lavage auto. Le contact direct de ces activités avec l'eau des lacs a entraîné une forte pollution causant des effets néfastes notamment le dégagement d'odeurs

\section{MATERIEL ET METHODES}

Milieu d'étude : L'étude a été effectuée sur huit (08) lacs du système lacustre de la ville de Yamoussoukro. Yamoussoukro est une ville du Centre de la Côte d'Ivoire (Figure 1). Yamoussoukro est la capitale politique du pays depuis 1983. Située entre les latitudes $6^{\circ} 48$ Nord et les longitudes $5^{\circ} 17$ Ouest, elle est le chef-lieu du département et de la région des lacs. Yamoussoukro est à $206 \mathrm{~km}$ d'Abidjan, la capitale économique. La ville de Yamoussoukro compte 207 000 habitants sur une superficie de $3500 \mathrm{~km}^{2}$. C'est la cinquième ville la plus peuplée du pays après Abidjan (4 707000 habitants), Bouaké (542000 habitants), Daloa (266 000 habitants) et Korhogo (245 000 habitants) (RGPH, 2014). La ville est située dans un nauséabondes, une perturbation de l'équilibre écologique du milieu naturel et un risque sanitaire par contamination microbiologique ou parasitaire (Diop, 1994 ; Khallaayoune, 2005 ; Sawadogo et al., 2005). Une étude axée sur l'évaluation de la contamination parasitologique des effluents domestiques à la station d'épuration de Cambérène (Dakar) par Akpo et al. (2013) a montré que les eaux usées des zones tropicales sont chargées en œufs et en kystes de parasites intestinaux. L'étude menée par Kouakou et al. (2014) sur les lacs de Yamoussoukro s'est limitée à la caractérisation chimique des sédiments sans toucher l'aspect parasitologique. La caractérisation parasitologique des eaux usées doit faire l'objet d'une attention particulière avant tout projet de réutilisation selon l'OMS (1997). L'objectif fixé dans ce présent travail est de faire la caractérisation parasitologique des lacs de Yamoussoukro ; afin de prévenir les populations et d'attirer l'attention des autorités quant aux risques liés à la pollution et à la réutilisation à l'état brut de ses eaux lacustres.

II s'agit de façon spécifique :

- d'identifier les différents types de parasites (helminthes et protozoaires) présents dans les eaux des lacs ;

- d'évaluer la charge parasitaire de ces eaux.

relief plat recouvert d'une savane arborée possédant un climat de type tropical humide de transition et est dotée d'un ensemble de lacs artificiels peu profonds aménagés sur deux cours d'eaux, qui communiquent entre eux (Figure 2). Les populations utilisent généralement des systèmes d'assainissement individuel par puits perdus ou fosses septiques dont certains sont en contact direct avec les réseaux d'évacuation d'eaux usées. Le rejet, sans traitement préalable, des effluents de diverses origines (domiciles, industries et ruissellements) dans les lacs à travers les canaux d'évacuation d'eaux usées (caniveaux et égouts) est une pratique accentuée dans la ville. (Kollia, 1998) 


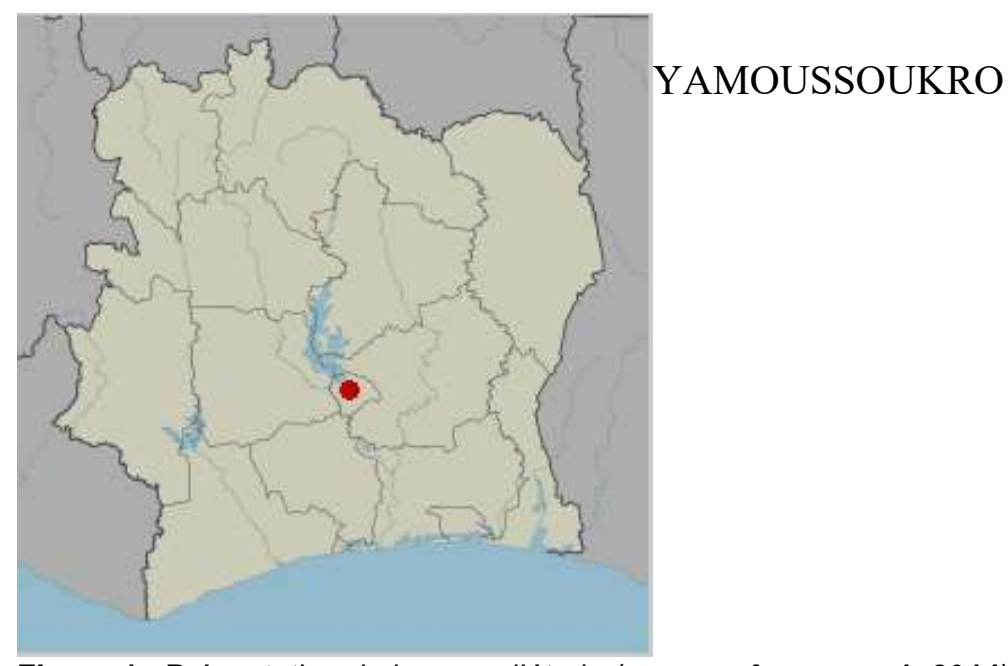

Figure 1 : Présentation de la zone d'étude (source : Anonyme 1, 2014)

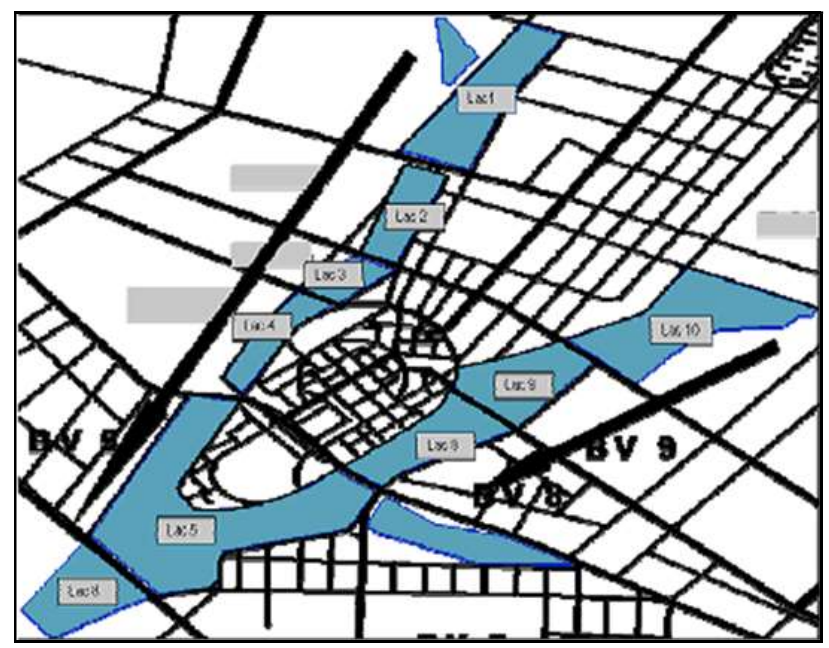

Figure 2: Présentation des lacs échantillonnés dans le système lacustre de Yamoussoukro (source : Kouakou et al., 2014)

Technique de collecte: L'eau a été prélevée manuellement dans des flacons stériles en polyéthylène d'une contenance de 1,5 litre préalablement secs et codifiés puis hermétiquement fermés. Au total, vingt-huit (28) stations réparties d'amont en aval couvrant l'ensemble des lacs de l'étude (G0, L1, L5, L6, L7, L8, L9 et L10) ont été visitées avec un nombre d'échantillon d'un litre (1L) par lac. II a été réalisé de deux (02) à sept (07) échantillons par lac (Tableau 1). Un volume de $5 \mathrm{~mL}$ d'alcool à $70^{\circ}$ est ajouté à chaque échantillon pour la fixation des microorganismes. Les flacons sont rangés dans des glacières puis transférés par la suite à l'Unité de Recherche en Génétique et Biologie Moléculaire de l'Université Jean Lorougnon Guédé pour les analyses parasitologiques. Pour une bonne estimation des résultats de l'étude, trois (03) campagnes de prélèvement allant de décembre 2015 à avril 2016 ont été effectuées. Ces campagnes de prélèvement se sont déroulées avec deux mois d'intervalle après chaque prélèvement, à savoir décembre-février-avril.

Technique d'analyse au laboratoire : Au laboratoire, les vingt-huit (28) échantillons d'eaux des lacs prélevés ont été analysés de décembre 2015 à avril 2016 par la technique de Bailenger modifiée (OMS, 1996). Cette technique a été utilisée pour la caractérisation parasitologique des échantillons car elle est la plus recommandée par l'OMS grâce à ces multiples avantages. Cette méthode permet la séparation des parasites par flottation et par sédimentation.L'observation microscopique des œufs d'helminthes a été basée sur la taille, la forme et le contenu de ces œufs en accord avec les descriptions bibliographiques (Golvan, 1985). L'identification des 
œufs d'helminthes a été effectuée au grossissement 400 tandis que les kystes de protozoaire en raison de leur petite taille ont été identifiés au grossissement 1000.

Pour plus de précision, on a répété la numération sur trois lames, et noté le nombre moyen d'œufs ou de kystes trouvé. Les parasites identifiés sont ensuite quantifiés. Le nombre total de parasites par litre $(\mathrm{N})$ d'eau collectée est calculé à l'aide de la formule suivante : $N=\frac{\text { A.X }}{\text { P.V }}$

Avec:

$N$ : nombre d'œufs ou de kystes par litre d'échantillon,

A : nombre moyen d'œufs ou de kystes comptés sur trois lames,

\section{RESULTATS ET DISCUSSION}

L'examen microscopique des échantillons d'eaux des lacs a permis de mettre en évidence treize (13) différents taxons de parasites. Tous les échantillons prélevés révèlent la présence de ces parasites sous la forme d'œufs ou de kystes appartenant respectivement aux groupes des helminthes et des protozoaires.

Caractérisation qualitative des parasites rencontrés: Parmi les parasites rencontrés ceux appartenant au groupe des helminthes se scindent en
$X$ : volume du produit final $(\mathrm{mL})$,

$P$ : Contenance de la lame $(0,15 \mathrm{~mL})$,

$V$ : volume de l'échantillon initial (en litre).

Analyses statistiques: Les données recueillies sur les charges parasitaires des lacs ont été soumises à des traitements statistiques. Ainsi, une analyse de variance (à un facteur) a été réalisée aux fins d'apprécier les différences entre les charges parasitaires des lacs. Lorsque la différence est révélée comme significative $(p<0,05)$, des tests de comparaisons multiples (Duncan) sont conduits. Ces traitements statistiques ont été effectués à l'aide du logiciel STATISTICA version 7.1.

deux classes, la classe des Nématodes qui comprend les taxons suivants: Ascaris sp, Trichuris sp., Strongyloïdes sp., Ankylostoma sp., Nematodirus sp., Toxocara sp., Ascaridia galli, Enterobius vermicularis et Capillaria sp. La classe des Cestodes est représentée par Tænia sp. et Hymenolepis diminuta. Les protozoaires recensés sont du genre Entamoeba. On a identifié aussi des larves de Strongles dans trois différents lacs (Figure 3).

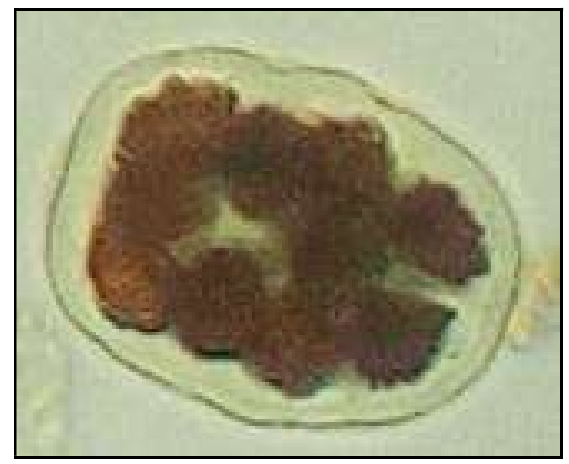

Kyste de Entamoeba

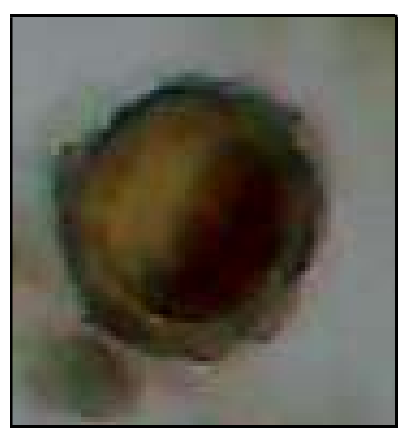

OEuf de Toxocara sp

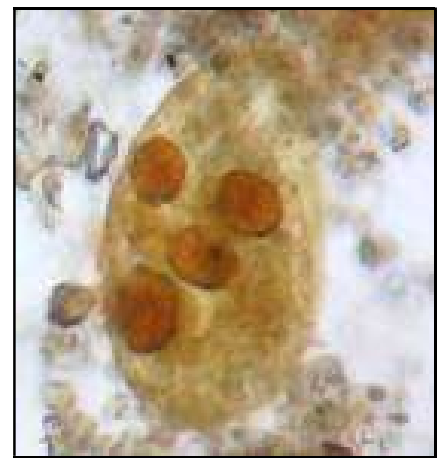

Kyste de Entamoeba

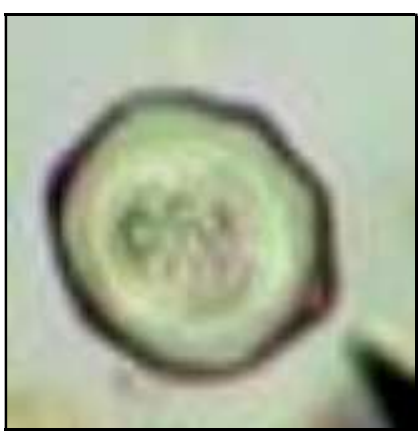

OEuf de Tænia sp

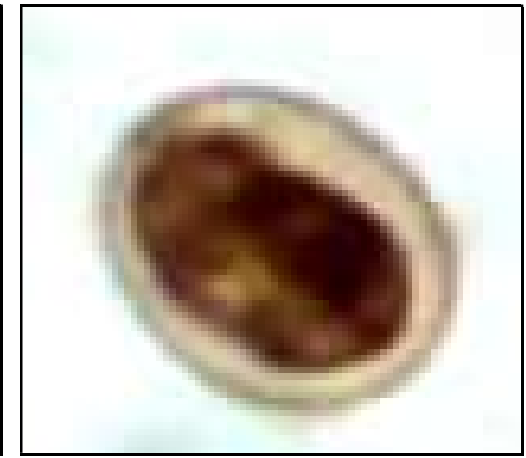

OEuf de Ankylostoma

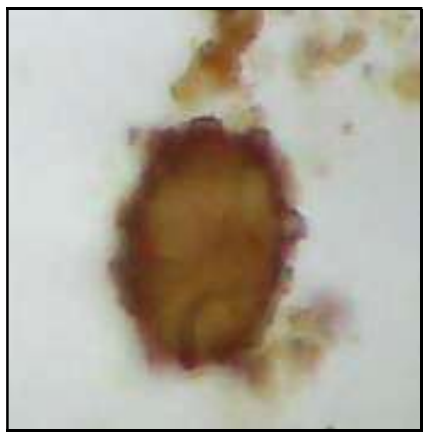

OEuf de Ascaris 


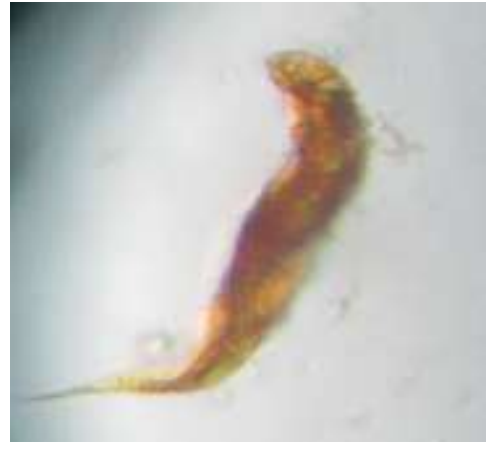

Larve de Strongles

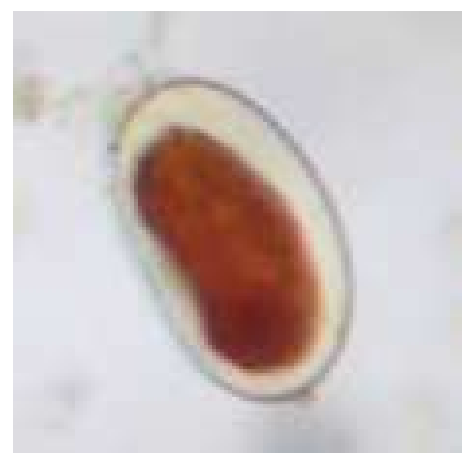

CEuf de Nematodirus sp.

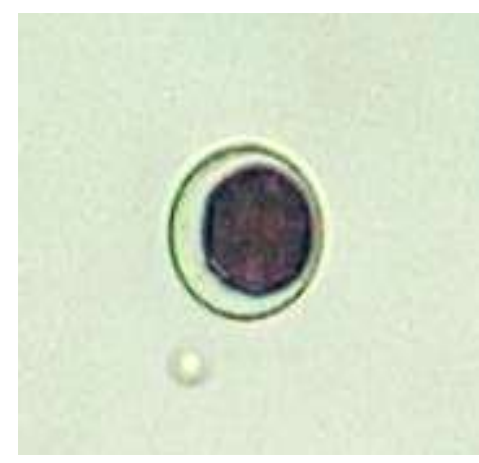

OEuf de Strongle

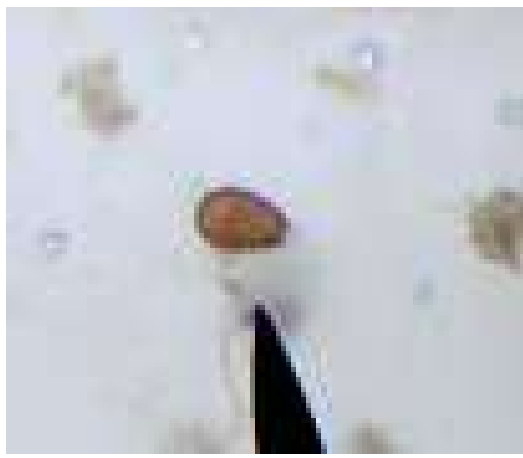

CEuf de Enterobius vermicularis

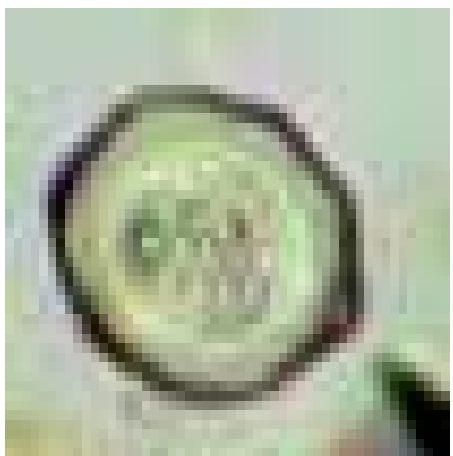

CEuf de Taenia sp.

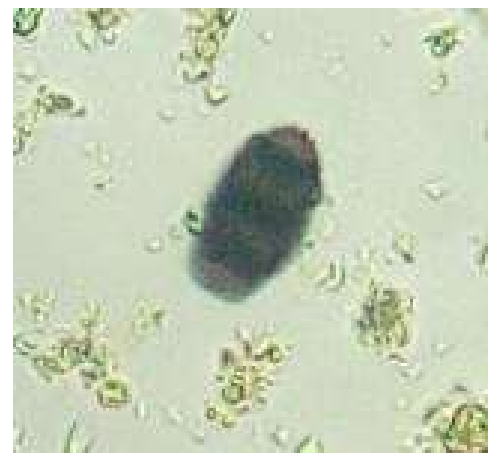

CEuf de Trichuris sp
Répartition et prévalences des parasites identifiés sur les lacs: Tous les lacs sont contaminés et le nombre de taxons par lac varie de 5 (Lac 7) à 12 (Lac 5). Les taxons parasitaires identifiées sont réparties différemment dans les lacs, certaines espèces sont présentes dans tous les lacs, d'autres par contre sont présentes dans quelques lacs; ce qui donne des prévalences inégales au niveau des lacs (Tableau 1). Ces prévalences partent de 38,5\% (Lac7) jusqu'à 92,3 $\%$ (Lac 5). En effet, le lac 5 (Lac 5) comporte 12 taxons sur les 13 rencontrés. II renferme plus de parasites avec une prévalence de $92,3 \%$. Cette prévalence est supérieure à celles des lacs $1 ; 6 ; 8 ;$ Go $; 9 ; 10$ et 7 qui sont respectivement de $69,2 \% ; 69,2 \% ; 69,2 \%$; $53,8 \% ; 53,8 \% ; 53,8 \%$ et $38,5 \%$ (Tableau 1). Sur l'ensemble des 13 taxons identifiés, huit (08) sont présents dans plus de $50 \%$ des lacs étudiés. On note la présence d'œufs de Ankylostoma sp. et de Strongles dans tous les lacs échantillonnés soit, $100 \%$ des lacs portent des œufs d'Ankylostomes et de Strongles, suivi des œufs de Ascaris sp. et des kystes de Entamoeba $s p$ qui sont présents à $87,5 \%$, puis viennent les œufs de Nematodirus sp., de Trichuris sp et des kystes de Entamoeba coli avec un taux de couverture de $75 \%$ chacun. Les espèces les moins recensées sont: Enterobius vermicularis (au niveau du Lac 1) et Hymenolepis diminuta (au niveau du Lac 9) avec des prévalences égales $(12,5 \%)$. Ces espèces se retrouvent seulement, chacune, dans un seul lac. Les larves de Strongles isolées ont une prévalence de 37,5 $\%$ (Tableau 2). 
Tableau 1: Répartition et prévalence des parasites identifiés dans huit lacs de la ville de Yamoussoukro (Côte d'Ivoire) de décembre 2015 à avril 2016

\begin{tabular}{|c|c|c|c|c|c|}
\hline Lacs & Nématodes & Cestodes & Protozoaires & Abondance & Prévalence (\%) \\
\hline G0 & $\begin{array}{l}\text {-Ankylostoma sp. } \\
\text {-Strongyloides sp. } \\
\text {-Ascaris sp, } \\
\text {-Trichuris sp. } \\
\text {-Toxocara sp. }\end{array}$ & & $\begin{array}{l}\text {-Entamoeba coli } \\
\text {-Entamoeba } \\
\text { histolytica. }\end{array}$ & 7 & 53,8 \\
\hline L1 & $\begin{array}{l}\text {-Ankylostoma sp. } \\
\text {-Strongyloides sp. } \\
\text {-Ascaris sp. } \\
\text {-Trichuris sp. } \\
\text {-Nematodirus sp. } \\
\text {-Ascaridia galli, } \\
\text { - Enterobius ver. }\end{array}$ & & $\begin{array}{l}\text {-Entamoeba coli } \\
\text {-Entamoeba } \\
\text { histolytica. }\end{array}$ & 9 & 69,2 \\
\hline L5 & $\begin{array}{l}\text {-Ankylostoma sp. } \\
\text {-Strongyloides sp. } \\
\text {-Ascaris sp. } \\
\text {-Trichuris sp. } \\
\text {-Toxocara sp. } \\
\text {-Ascaridia galli., } \\
\text {-Enterobius ver. } \\
\text {-Capillaria sp. } \\
\text {-Nematodirus sp. }\end{array}$ & -Tænia sp. & $\begin{array}{l}\text {-Entamoeba coli } \\
\text {-Entamoeba } \\
\text { histolytica. }\end{array}$ & 12 & 92,3 \\
\hline L6 & $\begin{array}{l}\text {-Ankylostoma sp. } \\
\text {-Strongyloides sp. } \\
\text {-Ascaris sp. } \\
\text {-Trichuris sp. } \\
\text {-Enterobius ver. } \\
\text {-Capillaria sp. } \\
\text {-Nematodirus sp. }\end{array}$ & & $\begin{array}{l}\text {-Entamoeba coli } \\
\text {-Entamoeba histolytica }\end{array}$ & 9 & 69,2 \\
\hline L7 & $\begin{array}{l}\text {-Ankylostoma sp. } \\
\text {-Strongyloides sp. } \\
\text {-Ascaris sp. } \\
\text {-Nematodirus sp } \\
\text {-Ascaridia galli. }\end{array}$ & & & 5 & 38,5 \\
\hline L8 & $\begin{array}{l}\text {-Ankylostoma sp. } \\
\text {-Strongyloides sp. } \\
\text {-Ascaris sp. } \\
\text {-Trichuris sp. } \\
\text {-Toxocara sp. } \\
\text {-Capillaria sp. } \\
\text {-Nematodirus sp. }\end{array}$ & & $\begin{array}{l}\text {-Entamoeba coli } \\
\text {-Entamoeba histolytica }\end{array}$ & 9 & 69,2 \\
\hline L9 & $\begin{array}{l}\text {-Ankylostoma sp. } \\
\text {-Strongyloides sp. } \\
\text { - Trichuris sp. }\end{array}$ & $\begin{array}{l}\text {-Tænia sp. } \\
\text {-Hymenolepis } \\
\text { diminuta }\end{array}$ & $\begin{array}{l}\text {-Entamoeba coli } \\
\text {-Entamoeba histolytica }\end{array}$ & 7 & 53,8 \\
\hline L10 & $\begin{array}{l}\text {-Ankylostoma sp. } \\
\text {-Strongyloides sp. } \\
\text { - Trichuris sp. } \\
\text {-Nematodirus sp. }\end{array}$ & -Tænia sp. & $\begin{array}{l}\text {-Entamoeba coli } \\
\text {-Entamoeba histolytica }\end{array}$ & 7 & 53,8 \\
\hline
\end{tabular}

L : lac G0 : lac Guiglo Enterobius ver: Enterobius vermicularis 
Tableau 2 : Prévalence de chacun des taxons de parasites isolés dans huit lacs de la ville de Yamoussoukro (Côte d'Ivoire) de décembre 2015 à avril 2016.

\begin{tabular}{c|c|c}
\hline \multicolumn{2}{c}{$(\mathrm{T}=8)$} & Prévalence en (\%) \\
\hline Taxons & $\mathbf{t}$ & 100 \\
Ankylostoma sp. & 8 & 100 \\
Strongyloïde sp. & 8 & 87,5 \\
Ascaris sp. & 7 & 75 \\
Nematodirus sp. & 6 & 75 \\
Trichuris sp. & 6 & 50 \\
Toxocara sp. & 4 & 25 \\
Capillaria sp. & 2 & 37,5 \\
Ascaridia galli & 3 & 12,5 \\
Enterobius vermicularis & 1 & 37,5 \\
Tænia sp. & 3 & 12,5 \\
Hymenolepis diminuta & 1 & 75 \\
Entamoeba coli & 6 & 87,5 \\
Entamoeba histolytica & 7 & 37,5 \\
Larve de Strongles & 3 &
\end{tabular}

$\mathbf{t}$ : nombre de lacs infestés $\quad \mathbf{T}:$ nombre total des lacs

Caractérisation quantitative des parasites: Quantitativement, les parasites recensés sont rencontrés dans des proportions différentes. Les plus nombreux sont les Nématodes, on dénombre neuf (9) espèces identifiées dans cette classe alors qu'on en compte que deux (2) chez les Cestodes ainsi que chez les Protozoaires; ce qui donne des parasites recensés $9 / 13$ soit $69,2 \%$ de Nématodes, $15,4 \%$ de Cestodes et $15,4 \%$ de Protozoaires (Figure 4). Sur l'ensemble des lacs, la charge parasitaire des espèces de parasites rencontrés varient de 0,66 œuf/litre à 123,97 œufs/litre. La plus forte charge est enregistrée au niveau de l'espèce Ankylostoma sp. avec une concentration moyenne de 123,97 œufs/litre et les plus faibles sont observées chez les espèces Hymenolepis diminuta et Capillaria sp. ayant des parasitémies moyennes respectives de 0,66 œuf/litre et de 1 œuf/litre (Figure 4). De plus, les concentrations des parasites rencontrés varient considérablement d'un groupe de parasites à l'autre et aussi en fonction des lacs. Pour les Nématodes, elles partent de 8,89 œufs/litre pour le lac
9 (L9) à 33,14 œufs/litre au niveau du lac 5 (L5), de 0 à 7,77 œufs/litre pour les Cestodes et 0 à 28,28 kystes/litre pour les Protozoaires. Les analyses statistiques montrent qu'il existe des différences significatives $(P<0,05 \%)$ entre les variations de la charge parasitaire des Nématodes $(P=0,03<0,05)$ et des protozoaires $(P=0,0001<0,05)$ en fonction des lacs mais celles des Cestodes ne présentent pas de différence significative dans les trois lacs où ils ont été rencontrés $(P=0,18>0,05)$ (Tableau 3$)$. Les résultats montrent aussi que les lacs sont plus contaminés par les helminthes parasites avec une concentration moyenne de 22,37 œufs/litre. Ces helminthes sont répartis en Nématodes et en Cestodes. Les œufs des Nématodes et des Cestodes ont des concentrations moyennes respectives de 20,17 œufs/litre et de 2,2 œufs/litre. Quant aux Protozoaires, ils présentent une concentration moyenne de 11,87 kystes/litre. Les Nématodes sont présents dans tous les lacs, les Cestodes dans trois (03) lacs et les Protozoaires dans sept (07) lacs (Figure 5). 


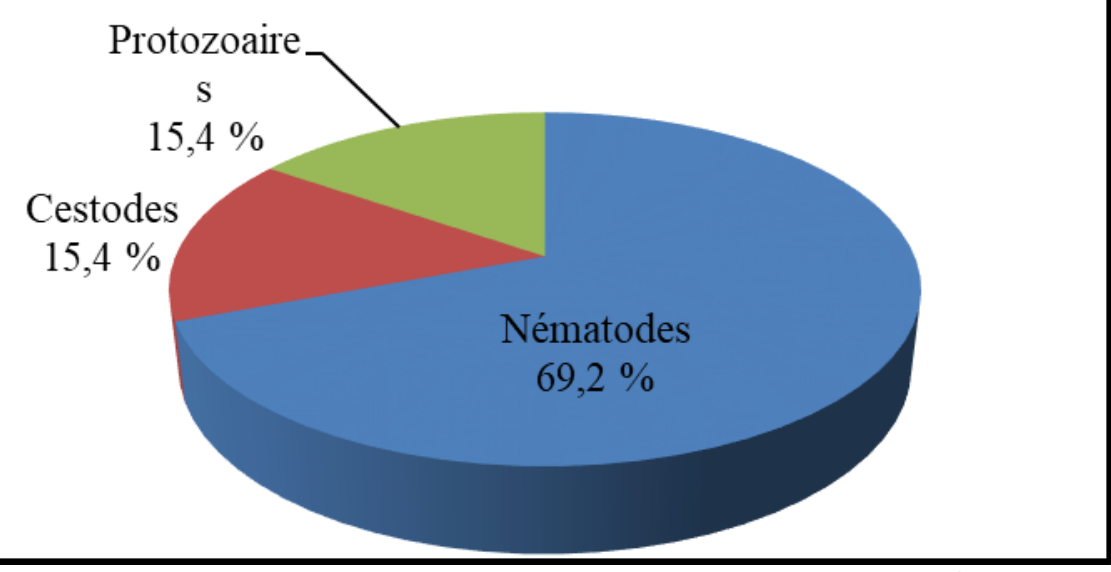

Figure 4 : Fréquences d'apparition des groupes de parasites identifiés dans huit lacs de Yamoussoukro (Côte d'Ivoire) de décembre 2015 à avril 2016

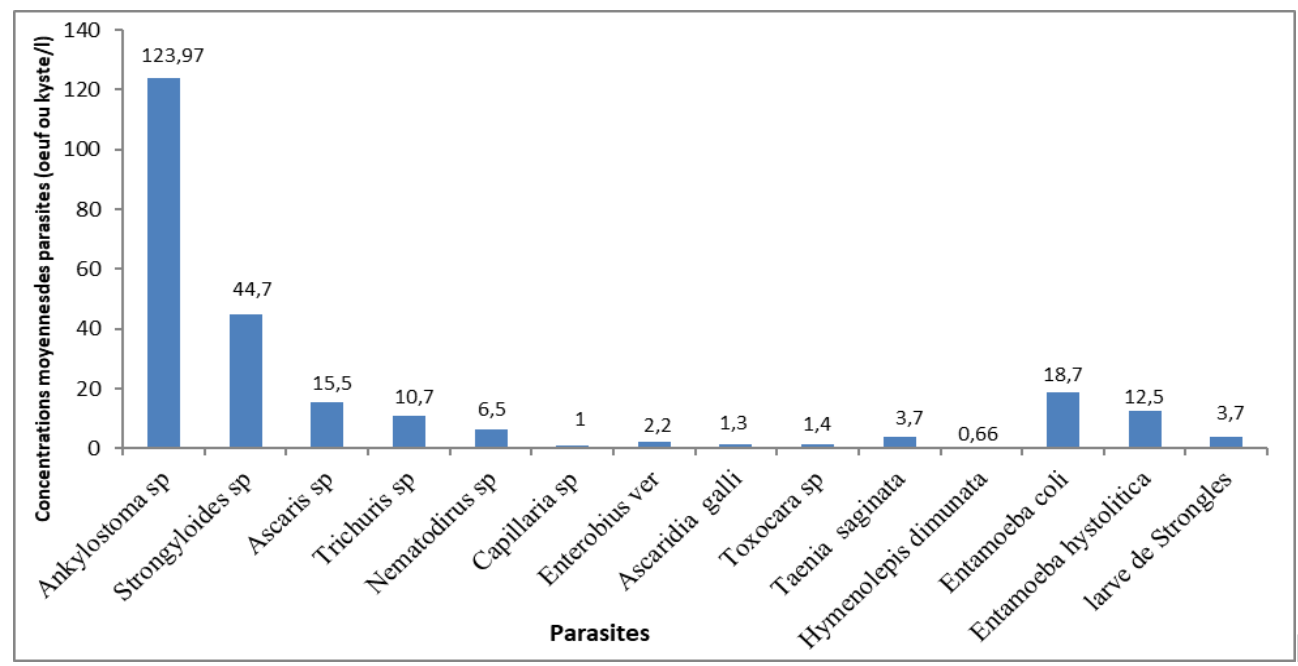

Concentrations moyennes des taxons de parasites identifiés dans huit lacs de la ville de Yamoussoukro (Côte d'Ivoire) de décembre 2015 à avril 2016.

Tableau 3 : Charge moyenne des groupes de parasites en fonction de huit lacs de la ville de Yamoussoukro (Côte d'Ivoire) de décembre 2015 à avril 2016

\begin{tabular}{|c|c|c|c|c|c|c|c|c|c|}
\hline \multirow[t]{2}{*}{ Groupes } & \multicolumn{8}{|c|}{ Charge parasitaire par lac (nombre d'œufs ou de kystes /litre) } & \multirow[t]{2}{*}{$p$} \\
\hline & G0 & $\mathrm{L} 1$ & L5 & $\mathrm{L} 6$ & L7 & L8 & L9 & L10 & \\
\hline $\begin{array}{c}\text { Nématode } \\
\mathrm{s}\end{array}$ & $\begin{array}{c}19,24^{\mathrm{a}} \pm \\
12\end{array}$ & $\begin{array}{c}22,16^{a} \pm 1 \\
2\end{array}$ & $\begin{array}{c}33,14^{a, b} \pm \\
21\end{array}$ & $\begin{array}{c}12,11^{\mathrm{a}, \mathrm{c}} \pm \\
10\end{array}$ & $\begin{array}{c}11,83^{\mathrm{a}, \mathrm{c}} \pm \\
10\end{array}$ & $\begin{array}{c}31,88^{a, b, d} \pm \\
16\end{array}$ & $\begin{array}{c}8,89^{\mathrm{a}, \mathrm{c}, \mathrm{e}} \\
\pm 5\end{array}$ & $22^{\mathrm{a}} \pm 12$ & 0,038 \\
\hline Cestodes & - & - & $6,65^{\mathrm{a}} \pm 10$ & - & - & - & $7,5 \pm 5^{\mathrm{a}}$ & $3^{a} \pm 7$ & 0,18 \\
\hline $\begin{array}{c}\text { Protozoair } \\
\text { es }\end{array}$ & $\begin{array}{c}5,25^{\mathrm{a}, \mathrm{b}} \pm \\
7\end{array}$ & $\begin{array}{c}13,15^{a, b} \pm \\
12\end{array}$ & $\begin{array}{c}28,28^{c} \pm 2 \\
1\end{array}$ & $\begin{array}{c}15,33^{a, b} \pm \\
12\end{array}$ & - & $\begin{array}{c}17,33^{\mathrm{a}, \mathrm{b}, \mathrm{c}} \mathbf{\pm} \\
15\end{array}$ & $7^{\mathrm{a}, \mathrm{b}} \pm 5$ & $\begin{array}{c}8,67^{a, b} \\
\pm 5\end{array}$ & $\begin{array}{c}0,000 \\
1\end{array}$ \\
\hline
\end{tabular}

- : pas d'œufs ou de kystes isolés

$a, b, c, d, e$ : les charges suivies de lettres différentes sur la même ligne diffèrent significativement au seuil de $5 \%$.

\section{DISCUSSION}

La caractérisation parasitologique des lacs de Yamoussoukro révèle une importante diversité spécifique et de charge parasitaire au niveau de chaque lac. Ceci concorde avec les résultats des travaux de Sylla et Belghyti (2008) dans l'agglomération urbaine de Sidi Yahia du Gharb (Maroc). Cette diversité peut être liée aux différents composants organiques que drainent les effluents bruts qui sont déversés dans 
les lacs. La diversité parasitaire a été aussi rapportée par d'autres auteurs qui ont signalé que les sources de contamination sont d'origines humaine et animale (Stot et al., 1997). L'analyse qualitative a permis de recenser treize (13) différents taxons de parasites sous forme d'œufs et de kystes appartenant à deux groupes de parasites intestinaux: - le groupe des helminthes au sein duquel on distingue deux classes; la classe des Nématodes représentée par Ascaris sp., Trichuris sp., Strongyloïdes sp., Ankylostoma sp., Nematodirus sp., Toxocara sp., Ascaridia galli, Enterobius vermicularis et Capillaria sp. et celle des Cestodes représentée par Tænia sp. et Hymenolepis diminuta. - le groupe des protozoaires est représenté par les espèces Entamoeba coli et Entamoeba sp. Ces données témoignent d'une pollution fécale véhiculée par les effluents bruts. Les helminthes sont les plus recensés avec une proportion de 84,6\% (11/13) par rapport aux protozoaires qui ne représentent que $15,4 \%$ de l'ensemble des parasites identifiés. La forte présence des helminthes a été aussi mise en évidence par Raweh et al. (2010) dans la ville de Kénitra au Maroc et cela serait lié à la croissance démographique qui augmente les quantités d'effluents bruts rejetés dans les eaux résiduaires (Bouhoum, 1996). La majorité des helminthes identifiés au cours de ces analyses appartiennent à la classe des Nématodes avec une fréquence de l'ordre de $81,8 \%$ contre $18,2 \%$ pour les Cestodes. Cette prédominance des œufs de Nématodes par rapport aux Cestodes a été également mise en évidence par d'autres travaux réalisés en Afrique notamment au Maroc (Abouelouafa, 2002; Bouhoum et al., 2002; Sylla et Belghyti, 2008), en Tunisie (Alouini et al., 1995) et ailleurs dans le monde, en France (Stien et Schwartzbrod, 1987). Cette prédominance peut s'expliquer par le fait que les œufs des Nématodes ont une plus grande résistance dans les eaux usées par rapport à ceux des Cestodes (Guessab et al., 1993; Alouini et al., 1995; Schwartzbrod et Banas, 2003). Les taxons recensés pendant nos analyses parasitologiques sont constitués de: Ascaris sp., Trichuris sp., Strongylö̈des sp., Ankylostoma sp., Nematodirus sp., Toxocara sp., Ascaridia galli, Capillaria sp., Enterobius vermicularis, Tænia sp., Hymenolepis diminuta, Entamoeba sp et Entamoeba coli. Huit (08) de ces taxons ont été également identifiés dans des travaux similaires effectués au Maroc par Sylla et Belghyti (2008). La confrontation des résultats de leurs travaux avec les nôtres donnent dans l'ensemble $61,5 \%$ de parasites en commun. Aussi $46,7 \%$ de résultats en commun avec les travaux El Guamri et Belghyti (2007) ; puis 53,8 \% de parasites en commun avec les travaux de Raweh et al. (2010) et $50 \%$ de parasites en commun avec ceux de Khallaayoune (2005). Cependant, une différence majeure de ces travaux aux nôtres est à souligner. En effet, dans nos travaux, le taxon Ankylostoma sp. est le parasite dont les œufs ont été le plus identifiés avec une distribution de $100 \%$ et une concentration moyenne de 123,97 œufs/litre sur l'ensemble des lacs tandis que les travaux antérieurs (El Guamri et Belghyti, 2007 ; Sylla et Belghyti, 2008 ; Raweh et al., 2010), ont relevé la présence d'un plus grand nombre d'œufs d'Ascaris $\mathrm{sp}$. dans leurs différents milieux. Cette différence pourrait s'expliquer par la composition organique des effluents qui serait différente du fait des habitudes culinaires ou par les pollutions liées aux modes de vie des populations (El Guamri et Belghyti, 2007) d'autant plus que nos travaux et ceux de ces auteurs se sont déroulés dans des environnements (pays) différents. Quantitativement, le niveau de la contamination (la charge parasitaire) varie considérablement d'un lac à l'autre, certains concentrent moins les parasites que d'autres. C'est le cas du lac 7 où on observe 11,83 œufs/litre, et des lacs 5 et 8 qui regorgent respectivement 33,14 et 31,88 œufs/litre comme charges des Nématodes. Les traitements statistiques indiquent qu'il existe des différences significatives $(P<$ $0,05)$ entre ces variations de la charge parasitaire. Ces différences peuvent être dues à la taille et du niveau socio-économique des populations urbaines raccordées à chaque lac (Jimenez et al., 2002; Nsom-zamo, 2003 ; Raweh et al., 2010). En effet, les lacs 5 et 8 sont situés en pleine ville et aux abords l'on constate l'implantation de certaines activités économiques telles les restaurants, les maquis, des hôtels, dont les effluents, les restes de nourriture et autres en provenance de ces lieux sont quotidiennement déversés dans ces lacs; surtout le lac 5 qui est un lac d'une grande étendue et raccordé à une population de grande taille où on observe en plus des activités implantées, certains endroits devenus des dépotoirs d'ordures ménagères et toilettes anarchiques à ciel ouvert. II est également la cible d'évacuation des effluents issus du centre hospitalier régional (CHR). Toutes ces réalités pourraient justifier sa forte charge parasitaire $(33,14$ œufs/litre et 28,28 kystes/litre) par rapport aux autres lacs moins vastes et en contact avec des populations de taille moyenne. Dans l'ensemble, les lacs de Yamoussoukro présentent une contamination en helminthes de 22,37 œufs/litre et de 11,87 kystes/litre en protozoaire. Les helminthes sont 
représentés par la classe des Nématodes et celle des Cestodes. Les concentrations moyennes en œufs de Nématodes et de Cestodes sont respectivement 20,17 œufs/litre et de 2,2 œufs/litre, comparées à celles obtenues par Khallaayoune (2005) dans quelques villes au Maroc, ces résultats révèlent des similarités et des discordances. Pour la classe des Nématodes, nos résultats se rapprochent de ceux obtenus par Khallaayoune dans la ville de Beni-Mellal (20,17 œufs/litre contre 18,2 œufs/litre) et identiques pour la classe des Cestodes obtenus à Marrakech (2,2 œufs/litre contre 2,2 œufs/litre). Par contre, les concentrations de Nématodes obtenues à Marrakech et à Ouarzazate (5,4 et 6,9 œufs/litre) sont inférieures aux nôtres, de plus la concentration des Cestodes à BeniMellal est largement supérieure (12,2 contre 2,2). Ces variations de la concentration des œufs d'helminthes pourrait s'expliquer par le fait que les conditions sociales, économiques, sanitaires et climatiques ne soient pas les mêmes dans les différentes villes où ont lieux ces travaux (Akpo et al., 2013) et aussi par la densité des populations raccordées aux eaux analysées (Raweh et al., 2010) ainsi que les quantités d'effluents que reçoivent ces eaux résiduaires. Concernant le groupe des protozoaires, les espèces recensées sous forme de kyste sont au nombre de deux, Entamoeba histolytica et de Entamoeba coli, avec une concentration moyenne de 11,87 kystes/litre. Ces deux espèces ont été également identifiées par Sylla et Belghyti en 2008 mais à une concentration plus faible de 4,84 kystes/litre. Les concentrations en œufs d'helminthes obtenues dans les échantillons d'eaux des

\section{CONCLUSION}

Au terme de cette étude consacrée à la caractérisation parasitologique des eaux des lacs de Yamoussoukro, on note, à travers les échantillons prélevés, que ces lacs renferment divers parasites. Les parasites mis en évidence appartiennent aux groupes des helminthes et des protozoaires dont les charges diffèrent considérablement d'un lac à l'autre. Les concentrations en œufs d'helminthes et en kystes de protozoaires obtenues dans les échantillons dépassent largement les normes recommandées par l'Organisation Mondiale de la Santé. Cette contamination parasitaire serait liée aux effluents urbains qui sont déchargés quotidiennement dans les lacs sans traitement préalable. Les activités agricoles pratiquées aux abords et aux environs de ces lacs notamment la pêche et le maraîchage qui nécessitent l'utilisation de l'eau des lacs sont supérieures aux normes recommandées par I'Organisation Mondiale de la Santé qui disent que la charge parasitaire doit être inférieure ou égale à un œuf viable par litre pour les eaux destinées à l'irrigation des cultures (Blumenthal et al., 2000). Cependant, les œufs des trématodes (Schistosoma sp., Clonorchis sinensis, Diphyllobothrium latum, Paragonimus sp., ) appartenant à la troisième classe des helminthes ne sont pas rencontrés lors de nos analyses. Ces résultats sont en accord avec ceux de El Guamri et Belghyti en 2003; Nsom-Zamo en 2003, à Kenitra ; Lahlou en 1992, dont l'étude a porté sur les eaux usées d'El Jadida (Maroc) et de ceux de Akpo et al. en 2013, sur les eaux usées de la station de Cambérène (Dakar). L'absence des œufs de cette classe serait liée soit à la densité de ces œufs (Akpo et al., 2013), soit à leur sédimentation et déformation rapide. Ces imperfections pourraient traduire les performances modestes de la méthode de Bailenger pour l'isolement des œufs de cette classe (OMS, 1997). Aussi, certains parasites appartenant aux protozoaires tels Giardia lamblia, Cryptosporidium parvum, Ixospora sp. ne sont pas rencontrés. II est donc clair que la méthode de Bailenger, bien qu'elle soit mondialement reconnue et conseillée par l'OMS grâce à ses avantages et performances, présente des limites du fait qu'elle n'arrive pas à mettre en évidence tous les types de parasites. D'autres méthodes de caractérisation parasitologique des eaux résiduaires telles la technique du SAF (Sodium Acétate-Acétique Formol) et celle de Kinyoung, sont donc envisageables pour ces types de parasites.

lacs, exposent les populations locales et environnantes ainsi que les animaux à un énorme risque sanitaire. Comme recommandations, nous proposons :

- la sensibilisation des populations quant aux risques liés à l'utilisation de l'eau des lacs et à la consommation des produits issus des maraîchages de la contrée ainsi que du poisson en provenance des lacs.

- rendre fonctionnelles les stations d'épuration d'eaux usées existantes afin d'éviter la contamination des lacs et les risques sanitaires des populations.

- aux autorités, l'entretien des lacs pour que ceux-ci soient caractéristiques de la capitale en lieu et place des odeurs et des maladies.

En perspective, nous comptons étendre cette étude à plusieurs villes du pays afin d'inciter les autorités administratives à prendre certaines initiatives telles que : 
- Prévenir les populations quant aux risques liés à la réutilisation des eaux usées brutes et à la consommation des produits issus de l'utilisation de ces eaux à travers l'organisation des campagnes de sensibilisation.

\section{REMERCIEMENTS :}

Les auteurs remercient Mme Malékah MOURADCONDE, Directeur Général de la Société de développement touristique de la région des lacs

\section{RÉFÉRENCES}

Abouelouafa M., 2002. Traitement et réutilisation des eaux usées en agriculture au Maroc Oriental (Oujda): Etude physico-chimique, agronomique et sanitaire. Thèse de Doctorat. Fac. Sci. Oujda (Maroc), 141p.

Akpo Y., Sawadogo G.J. et Degnon R.G., 2013. Évaluation de la contamination parasitologique des eaux usées domestiques à la station d'épuration de Cambérène (Dakar). Journal of Applied Biosciences, 69 : 5449 - 5455.

Alouini Z., Achour H. et Alouini A., 1995. "Devenir de la charge parasitaire des eaux usées traitées dans le réseau d'irrigation "Cebala" in Zekri, Laajini A." (Ed) agriculture durabilité et environnement. Zaragoza: CIHEAM, 117-124.

Amy G., Debroux J.F., Arnold R. and Wilson L.G., 1996. Preozonation for enhancing the biodegradability of wastewater effluent in a potable-recovery soil aquifer treatment (SAT) system. Rev. Sci. Eau, 9, pp 365-380.

Anonyme 1. 2014 https:// www.google.com/ search?q=carte + de + Yamoussoukro Consulté le 6 mars 2014

Attab S., 2011. Amélioration de la qualité microbiologique des eaux épurées par boues activées de la station d'épuration haoud berkaoui par l'utilisation d'un filtre à sable local. Mémoire Magister, faculté des sciences de la nature et de la vie et sciences de la terre et de l'univers. Option: microbiologie appliquée. Université Kasdi Merbah-Ouargla. Ouargla, Algérie, 152p.

Bailenger J., 1979. Mechanisms of parasitological concentration in coprology and their parasitical consequences. Journal of American Medical Technology, 41: 65-71.

Blumenthal UJ., Mara Duncan D., Peasey A., Guillermo R-P., and Stott R., 2000. Guidelines for the microbiological quality of treated
- Veiller à l'atteinte des recommandations de l'OMS par l'installation de stations de traitement des effluents bruts.

(SODERTOUR-LACS) pour l'hébergement et la restauration lors de cette étude à Yamoussoukro.

wastewater used in agriculture: recommendations for revising WHO guidelines. Bull of the World Health Organization, 78(9):11. 04-16.

Bontoux, 1993. Introduction à l'étude des eaux douces : eaux naturelles, eaux usées, eaux de boisson. Edition Technique et Documentation Lavoisier, pp 166.

Bouhoum K. and Schwartzbrod J., 1989.Quantification of helminth eggs in wastewater. Zentralblatt für Hygiene und Umweltmedizin, 188: 322-330.

Bouhoum K., 1996. "Etude épidémiologique des helminthiases intestinales chez les enfants de la zones d'épandage des eaux usées de Marrakech / Devenir des kystes de protozoaires et des œufs d'helminthes dans les différents systèmes extensifs de traitement des eaux usées" Thèse Doct. Univ. Marrakech, 227p.

Bouhoum K., Amahmid O., Asmama S., 2002. "Wastewater reuse for agricultural purposes: Effects on population and irrigated crops" Proceeding of international symposium environnmental pollution control and waste management. EPCOWM.Tunis, Part II. P: 582586.

Diop BS., 1994. Traitement des eaux usées domestiques par voie naturelle, sous climat tropical. Mémoire de DEA Environnement, ISE : Dakar ; 322p.

El Guamri Y. et Belghyti D., 2007. Charge parasitaire des eaux usées brutes de la ville de Kénitra (Maroc) Afrique SCIENCE 03(1) 123 - 145.

Guessab M., Bize J., J. Schwartzbrod, A. Mani, M. Morlot, N. Nivault, Schwartzbrod L., 1993. "Wastewater traetement dry infiltration percolation on sand: results in Ben Sergao. Morocco" Wast. Sci. Tech., 17 91-95. 
Golvan J., 1985. «Les nouvelles techniques de parasitologie». Ed. Flammarion MédecineSciences, 67p.

Jimenez B., Maya C., Sanchez E., Romero A., Lira L., Barrios J.A., 2002. "Comparison of the quantity and quality of the microbiological content of sludge in countries with low and high content of pathogens" Water. Sci. Tech., 46p

Kazi, T. G., Arain, M. B., Jamali, M. K., Jalbani, N., Afridi, H. I., Sarfraz, R. A., Baig, J. A., Shah, A. Q., 2009. Assessment of water quality of polluted lake using multivariate statistical techniques: A case study. Ecotoxicology and Environmental Safety 72, 301-309.

Khallaayoune K., 2005. Epidémiologie des maladies parasitaires liées aux eaux usées : situation au Maroc et stratégies de prévention (4) in compte rendu Atelier- séminaire sur traitement et réutilisation des eaux usées : impact sur la santé et l'environnement du 3-6 mars. Rabat: IAV. 14p.

Kolia C., 1998. Influence réciproque de la qualité des eaux naturelles et de la présence des végétaux aquatiques. Mémoire fin de cylegénie alimentaire.

Kouakou K. A., Aw S., Adamou M. M., Siaka S., Savane I., 2014. Caractérisation des sédiments du système lacustre de Yamoussoukro (Côte d'Ivoire) et spéciation de leurs phosphores (Characterization of sediments of Yamoussoukro lake system (Côte d'Ivoire) and their phosphorus speciation), Yamoussoukro (Côte d'Ivoire), J. Mater. Environ. Sci. 5 (4) (2014), 1013-1020.

Nsom-zamo A.-CL., 2003. Pollution par les eaux usées: Analyse parasitologique des eaux usées brutes et des cultures maraîchères, étude de la viabilité des helminthes et évaluation du risque sanitaire humain dans la région $\mathrm{du}$ Gharb (Kenitra). Thèse de Doctorat National en Parasitologie. Faculté des Sciences de Kenitra, Maroc, 160p.

Nsom-zamo A.-C, Belghyti D., Lyagoubi M., 2003. Parasitological study of helminthes eggs carried by the untreated wastewater of the Maamora urban district, Kénitra-Morocco, Journal Européen d'Hydrologie, tome 34, fasc. 2, (2003b) 245-250.

OMS, 1989. L'utilisation des eaux usées en agriculture et aquaculture : Recommandations à visées sanitaires. Organisation Mondiale de la Santé, Genève, pp 17-60.

OMS, (1997). "Analyse des eaux résiduaires en vue de leur recyclage en agriculture" Manuel des techniques de laboratoire en parasitologie et bactériologie, Genève. 31p.

Raweh S., Elkharrim K., Cissé M., El Guamri Y., Abchir Y., Belghyti D., 2010. Aspect parsitologique des eaux usées rejetees dans le bas Sebou (Kenitra, Maroc) World Journal of Biological Research 003: 1. 12p.

Récensement Général de la Population et de l'Habitat (RGPH) 2014. Institut National de la Statistique de Côte d'Ivoire.

Sadat A.W., N'goran E. B. Z., Sorho S., Parinet B., 2011. Intérêt de l'analyse multidimensionnelle pour l'évaluation de la qualité physicochimique de l'eau d'un système lacustre tropical : cas des lacs de Yamoussoukro (Côte d'Ivoire). Journal of Applied Biosciences 38: $2573-2585$.

Sawadogo G.J., Téko-agbo A., Akpo Y., 2005. Réutilisation des eaux usées en agriculture au Sénégal : impact sur la santé et l'environnement in compte rendu Atelierséminaire sur traitement et réutilisation des eaux usées : impact sur la santé et l'environnement du 3-6 mars. Rabat: IAV. - 14 p.

Schwartzbrod J. and Banas S., 2003. "Parasite contamination of liquid sludge from urban wastewater traetment plants" Wat. Sci. Tech., Vol. $47 \mathrm{~N}^{\circ} 3$. pp 163-166.

Stien J.L. et Schwartzbrod J., 1987. "Devenir des œufs d'helminthes au cours d'un cycle d'épuration des eaux usées urbaines" Revue internationale des séries de l'eau, 3 (3/4): 77 82.

Stot R., Jenkins T. and Shabana M., 1997. May "A survey of the microbial quality of wastewater in Ismailia, Egypt and the implications for wastewater reuse" Wat. Sc. Tech. 35 (11-12) 211-217.

Strobl, R.O. and Robillard P.D., 2008. Network design for water quality monitoring of surface freshwaters: A review. Journal of Environmental Management 87, 639-648.

Sylla I. et Belghyti D., 2008. Analyse parasitologique des eaux usées brutes de la ville de Sidi Yahia du Gharb (Maroc). World Journal of Biological Research. ISSN: 1994-5108. ISPROMS, 10p. 